\title{
Study on Out-of-step Oscillation Based on Oscillation Energy Flow
}

\author{
Yanbo Chen ${ }^{1}$, Yangzi Wang ${ }^{1}$, Jin $\mathrm{Ma}^{2}$ \\ ${ }^{1}$ School of Electrical and Electronic Engineering, North China Electric Power University, Beijing, 102206, China \\ ${ }^{2}$ School of Electrical and Information Engineering, University of Sydney, NSW, 2006, Australia
}

\begin{abstract}
With the scale of interconnected power systems becoming larger and larger, asynchronous oscillation occurs increasingly frequently. Under this circumstance, the identification of out-of-step oscillation along with corresponding separation strategy plays a vital role in preventing catastrophic black outage caused by spreading accidents. During out-of-step oscillation process, electric quantities vary so rapidly and dramatically that traditional methods of oscillation analysis, like small signal analysis, are no longer appropriate in this scenario. In this paper, direct method is used to study the dynamic characteristics of outof-step oscillation. Based on Dirac structure and port Hamilton, energy structure is constructed and extended to electric power system. Then oscillation energy flow is derived and used in further research on the electrical characteristics' variation rules during out-of-step oscillation process. With analytic functions in the equivalent twogenerator system, the problem of out-of-step oscillation is expounded in terms of oscillation energy flow on the tie line.
\end{abstract}

Keywords-out-of-step oscillation; direct method; Energy structure; oscillation energy flow.

\section{INTRODUCTION}

For a physical system, scale and complexity always come hand in hand. Electric power system is as well. Under these circumstances, a little accident could cause cascading events that lead to destructive consequences. As an indispensable part of the third defensive line of power system, the identification of out-of-step oscillation and separation strategy shoulder great responsibility in securing its safety and reliability. When out-of-step oscillation occurs, splitting the whole system into several subsystems with different frequency from the out-of-step center can not only guarantee the stability of subsystems, but also avoid further damaging impacts.

Most of the current modelling approaches of physical systems are based on some sort of network representation, where the physical system under consideration is seen as the interconnection of a large number of simple subsystems with ports [1]. These means of modelling possess many advantages. The most obvious one is that we can study their interaction by observing their port where they interact. Power system is a large complex system and oscillation is a specific kind of interaction. Motivated by this, port Hamilton and Dirac structure are joined together to construct the energy structure of power system. Based on this, oscillation energy flow on port is derived for further observation.

The rest of the paper is organized as follows: the second section gives a brief introduction of Dirac structure, port Hamilton and energy structure; in the third section, out-of-step oscillation is studied with oscillation energy flow; section four concludes this paper.

\section{OSCILLATION ENERGY FLOW}

\section{A. Dirac structure}

Dirac structures are used to mathematically formalize the power-conserving interconnection of physical system. Let $F$ and $E$ be real vector spaces whose elements are labeled as $f$ and $e$, respectively. We call $F$ the space of flows and $E$ the space of efforts. The space $B=F \times E$ is called the bond space and an element of the space is denoted by $b=(f, e)$. The spaces $F$ and $E$ are power conjugate. This means that there exists a map

$$
\langle\cdot \mid \cdot\rangle: E \times F \rightarrow R
$$

Called the power product such that it is linear in each coordinate and it is not degenerate. Using the power product we define a symmetric bilinear form

$$
<<\left(f^{a}, e^{a}\right),\left(f^{b}, e^{b}\right)>>:=<e^{a}\left|f^{b}>+<e^{b}\right| f^{a}>
$$

Let $D$ be a subspace of $B$. $D$ is a Dirac structure if $D=D^{\perp}, D^{\perp}$ is the orthogonal complement of $D$ with respect to the bilinear form above. Dirac structure not only follows the energy conservation law, but the composition of two Dirac structures is again a Dirac structure according to [2, 3].

\section{B. Port Hamilton}

$$
\left\{\begin{array}{l}
\dot{x}=J(\mathrm{x}) \frac{\partial H(\mathrm{x})}{\partial x}+G(\mathrm{x}) f \\
\dot{e}=G^{T}(\mathrm{x}) \frac{\partial H(\mathrm{x})}{\partial x}
\end{array}\right.
$$

Here $X$ denotes the vector of energy variables. $H(x)$ is the total stored energy of the sub-system, with $J(x)$ denoting the transposed gradient vector of $H$, and the $n \times n$ structure matrix $J(x)$ is associated with the network topology of the observed subsystem. Port Hamilton provides mathematical description for the energy storing function. 


\section{Energy structure}

In general, a system is composed of four kinds of dynamic components: components of energy supply, energy consuming, energy storing and energy exchange.
With Dirac structure and Port Hamilton, the energy structure of a normal subsystem could be modelled as Fig.1.

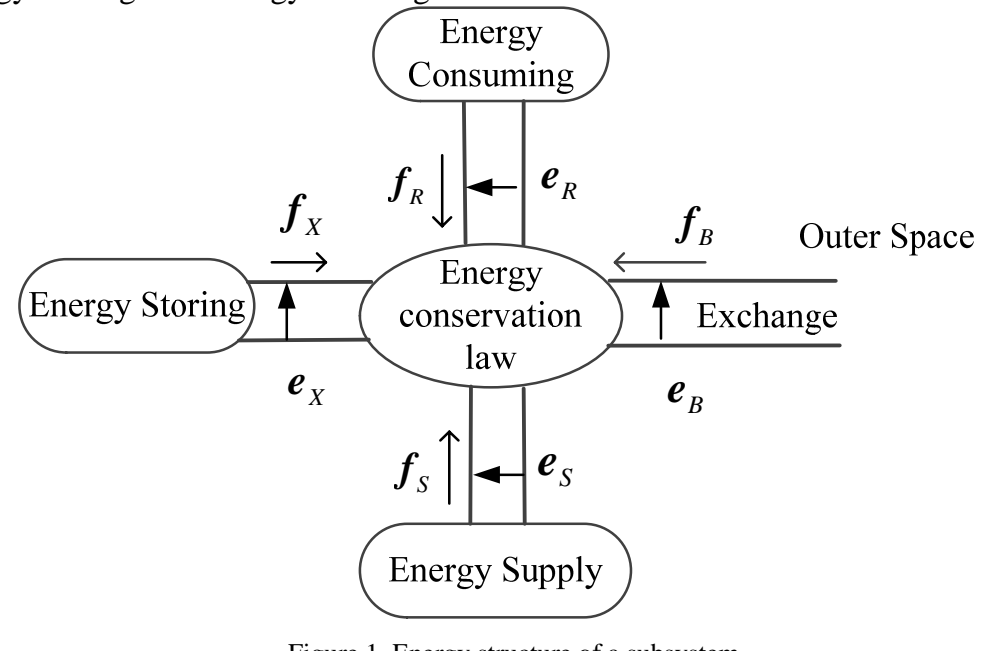

Figure 1. Energy structure of a subsystem

For a system of $n$ buses, of which $n_{G}$ are generator buses, the energy on the energy structure is as (4) [4]. And it is strictly conformed to Lyapunov energy function [5].

$$
\begin{aligned}
& V=\sum_{i=1}^{n_{G}} V_{i, \text { energy storing }}-\sum_{i=1}^{n_{G}} V_{i, \text { energy sup ply }}-\sum_{i=1}^{n_{G}} V_{i, \text { energy exchange }} \\
& +\sum_{i=n_{G}+1}^{n} V_{i, \text { energy storing }}-\sum_{i=n_{G}+1}^{n} V_{i, \text { energy exchange }} \\
& =\left(-\frac{1}{2} \sum_{i=1}^{n} B_{i i} U_{i}^{2}-\sum_{i=1}^{n-1} \sum_{j=i+1}^{n} U_{i} U_{j} B_{i j} \cos \theta_{i j}\right)+ \\
& \left.\sum_{i=1}^{n_{G}} \frac{1}{2} \frac{1}{2} \frac{E_{q i}^{\prime 2}}{x_{d i}-x_{d i}^{\prime}}+\frac{1}{2} \frac{E_{d i}^{\prime 2}}{x_{q i}-x_{q i}^{\prime}}+\frac{1}{2} \omega_{0}^{\prime} \omega_{i}^{2} I_{d i}^{2}+\int_{\delta_{i 0}}^{\delta_{i}} P_{m i} d \delta_{i}-\int_{E_{q i}^{\prime}}^{E_{q i}} \frac{E_{f i} I_{q i}^{2}}{X_{d i}-x_{q i}^{\prime}}\right) \\
& -\sum_{i=n_{G}}^{n}\left(\int_{\theta_{i 0}}^{\theta_{i}} P_{i} d \theta_{i}+\int_{U_{i 0}}^{U_{i}} Q_{i} \frac{d U_{i}}{U_{i}}\right)
\end{aligned}
$$

In (4), generators are described by $4^{\text {th }}$-order models and the loads are treated as constant power. $\mathrm{V}$ denotes the energy on the energy structure. $\mathrm{B}$ is the reactance matrix of the network; $\mathrm{U}$ is the magnitude of bus voltage; $\theta$ is phase angle of bus voltage; $\delta$ is power angle; $\omega$ is the angular velocity; $E_{f}$ is excitation voltage ; $E_{q}^{\prime}$ is the $\mathrm{q}$ axis transient electromotive force and $E_{d}^{\prime}$ is the d axis transient electromotive force; $M$ is the inertia time constant of generators; $x_{d}, x_{q}, x_{d}^{\prime}, x_{q}^{\prime}$ are synchronous reactance and transient reactance of $\mathrm{d}$ axis and q axis; $P_{m}$ is the mechanical power; $P$ is real power and $Q$ is reactive power.

There are three points worth noting:

1) Energy accumulated on the system symbolizes how stable the system is. The more energy the observed subsystem accumulates, the easier it will lose stability [6].

2) If we consider energy supply as a specific kind of energy exchange, the energy accumulated on the subsystem is directly and only be determined by energy exchange.

3) If energy exchange is positive, it means energy is being transmitted to the observed subsystem and will excite oscillation. If energy exchange is negative, it means energy is being transmitted from the observed subsystem. Oscillation amplitude will be dampened and stability will again be restored.

Because energy exchange is closely connected to oscillation, we define the energy exchange, i.e. the last term of (4) as oscillation energy flow (OEF)

$$
O E F(t)=\int_{\theta_{0}}^{\theta} P d \theta+\int_{U_{0}}^{U} Q \frac{d U}{U}
$$

\section{STUDY ON OUT-OF-STEP OSCILLATION BASED ON $\mathrm{OEF}$}

As the oscillation occurs on the tie line interconnecting different subsystems, we should extend our range of observation from a single port to the whole tie line.

In general, when out-of-step oscillation happens in a large system, all the generators could be divided into two 
coherent groups approximately. It's appropriate to use equivalent two-generator system to illustrate the out-of- step oscillation process. Fig.2 shows the wiring diagram of equivalent two-generator system.

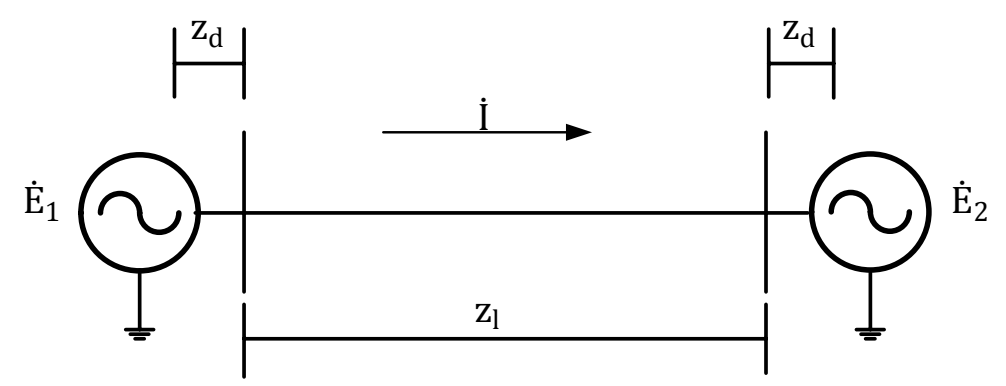

Figure 2. Wiring diagram of equivalent two-generator system

Electric potentials on the two sides of the tie line is $\dot{E}_{1}$, $\dot{E}_{2}, \dot{E}_{1}=E_{1} \angle \delta$ and $\dot{E}_{2}=k E_{1} ; \delta$ is equivalent power angle difference between the two equivalent systems on the two ends; $k$ is voltage magnitude ratio; Positive sequence equivalent impedance of equivalent generator is $z_{d}$, impedance of the tie line is $Z_{l}$. Assume the system impedance angle is equal to the line impedance angle and the impedance on the tie line is evenly distributed. Then all the impedance is aggregated to $z_{\Sigma}=z_{d}+z_{d}+z_{l}$.Assume electric current $I$ flows from node 1 to node 2 .

The electric distance of the point of concern on the tie line from node 1 is $z=r+j x$. Assume $\rho=z / z_{\Sigma}$, $\varphi=\arctan (x / r)$. Voltage of location of concern on the tie line could be calculated as follows

$$
\begin{aligned}
& \dot{U}=\dot{E}_{1}-z \dot{I}=\dot{E}_{1}-\frac{Z}{Z_{\Sigma}}\left(\dot{E}_{1}-\dot{E}_{2}\right) \\
& =E_{1}[(1-\rho) \cos \delta+k \rho+j(1-\rho) \sin \delta]
\end{aligned}
$$

When $\delta=180^{\circ}$, we have

$$
|U|_{\min }^{2}=k \rho-1+\rho
$$

Obviously, on the location $\rho=1 /(1+k)$, the minimum value of voltage magnitude is zero at this moment. This location on the tie line is the out-of-step center of this oscillation period. Take line loss into consideration,

$$
\begin{aligned}
& \Delta S=\frac{P^{2}+Q^{2}}{E_{1}^{2}}(r+j x) \\
& =\frac{\left(1+k^{2}-2 k \cos \delta\right) E_{1}^{2}}{\left|z_{\Sigma}\right|} \rho(\cos \varphi+j \sin \varphi)
\end{aligned}
$$

From (4) (5) (7), oscillation energy flow could be calculated as,

$$
O E F(t)=\left.Q \ln U\right|_{U_{0}} ^{U}+\left.P \theta\right|_{\theta_{0}} ^{\theta}
$$

In order to figure out how it varies during an oscillation period, Taylor's series expansion is taken advantage of, with terms involving second and higher order powers of $\Delta \delta$ neglected. In a very short period of time $\Delta t$, we linearize (8) and get,

$$
\begin{aligned}
& O E F(\mathrm{t})=\int_{0}^{t}\left(\mathrm{P} \dot{\theta}+\frac{Q}{U} \dot{\mathrm{U}}\right) d t \\
& =\frac{k E_{1}^{2}}{\left|z_{\Sigma}\right|} \sin \delta \cdot \frac{(1-\rho)^{2}+k \rho(1-\rho) \cos \delta}{k^{2} \rho^{2}+(1-\rho)^{2}+2 k \rho(1-\rho) \cos \delta} \cdot \Delta \delta+ \\
& \frac{E_{1}^{2}}{\left|z_{\Sigma}\right|}\left[1-\rho-\rho k^{2}+k(2 \rho-1) \cos \delta\right] \cdot \frac{k(\rho-1) \rho \sin \delta}{k^{2} \rho^{2}+(1-\rho)^{2}+2 k \rho(1-\rho) \cos \delta} \cdot \Delta \delta \\
& =\frac{k E_{1}^{2}}{\left|z_{\Sigma}\right|}(1-\rho) \sin \delta \cdot \Delta \delta
\end{aligned}
$$

It could be seen that oscillation energy flow on every point of the tie line changes following a sinusoidal curve in each oscillation period. Fig.3 and Fig.4 show the simulation cases on equivalent two-generator system. From Fig.3 and Fig.4 we could see: 
1) In one out-of-step oscillation period, oscillation energy flow transmitted from side 1 changes from positive to negative. It means when $\delta \in\left(0^{\circ}, 180^{\circ}\right)$, energy accumulated in System 1 decreases while energy accumulated in System 2 increases; when $\delta \in\left(180^{\circ}, 360^{\circ}\right)$, energy accumulated in System 1 increases while energy accumulated in System 2 decreases.

2) It is apparent to see that when $\delta$ approaches to $180^{\circ}$, there are two peaks of oscillation energy flow values lying in the middle of the tie line ( $\rho=1 / 2$ ), the out-of-step center. The peak lies near System 1 is positive: the oscillation energy flow there is being transmitted from System 1 to System 2; while the peak lies near System 2 is negative: the oscillation energy flow there is being transmitted from System 2 to System 1. It means in an oscillation period, when equivalent power angle difference $\delta$ reaches $180^{\circ}$, oscillation energy flows are transmitted from both ends of the tie line to the out-ofstep center and converge there.

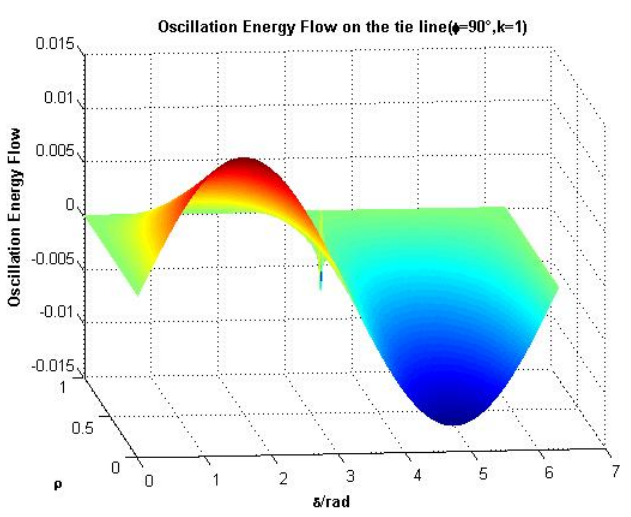

Figure 3. Oscillation energy flow ( $\varphi=90^{\circ}, k=1$ )

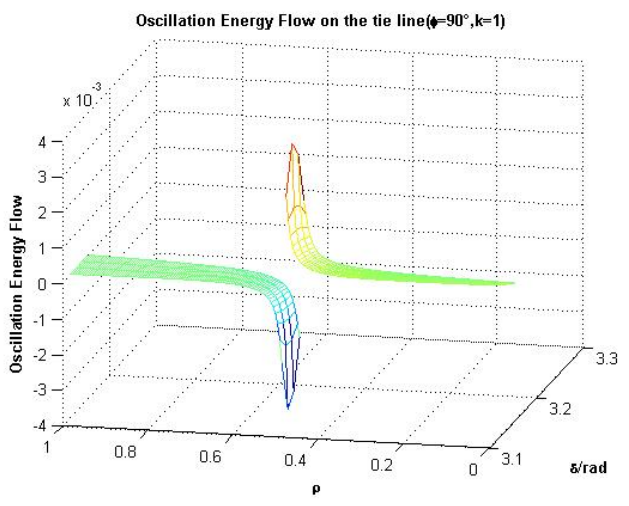

Figure 4. Oscillation energy flow ( $\varphi=90^{\circ}, k=1$ ) on the out-of-step center

\section{CONCLUSION}

This paper analyzes the dynamic behavior between subsystems during out-of-step process from the perspective of oscillation energy flow. When out-of -step oscillation occurs, oscillation energy flow on the tie line varies follows a sinusoidal curve and two peaks could be found on the out-of-step center. When the peaks are found on the tie line, it means out-of-step oscillation has occurred and splitting devices should be informed. Our future work will focus on the splitting criterion based on the dynamic characteristics oscillation energy flow demonstrates.

\section{ACKNOWLEDGEMENTS}

This work was supported in part by State Grid Corporation of China, Major Projects on Planning and Operation Control of Large Scale Grid (SGCCMPLG019-2012) and in part by the National High Technology Research and Development Program (2012AA050208).

\section{REFERENCES}

[1] Van der Schaft A. J., Dalsmo M., Maschke B. M., Mathematical structures in the network representation of energy-conserving physical systems. Proceedings of the $35^{\text {th }}$ Conference on Decision and Control: Vol.1, 1996, Kobe, Japan: 201-106.

[2] Carles Batlle, Imma Massana, Ester Simo, Representation of a general comparison of Dirac structures. 50th IEEE Conference on Decision and Control and European Control Conference (CDCECC):Vol.1,12-15 Dec 2011,Orlando,FL, USA:5199-5204.

[3] Cervera J., Van der Schaft A. J., Banos A., Interconnection of PortHamiltonian Systems and Composition of Dirac Structures. Automatica, 2005(43): 212-225.

[4] Li Ying. Methodology for locating the oscillation sources in power systems based on the energy structure. Tsinghua University.

[5] Min Yong, Chen Lei. A transient energy function for power systems including the induction motor model. Science in China Series E: Technological Sciences, VOL.50, No.5, pp. 575-584, Oct. 2007.

[6] N. A. Tsolas, A. Arapostathis, P. P. Varaiya, A structure preserving energy function for power system transient stability analysis. IEEE Transaction on Circuits and System, vol. cas-32, No.10, pp: 10411049, Oct. 1985.

[7] Kundur P., Power Systems stability and control. New York: McGraw-Hill, 1994. 